\title{
Medium induced gluon spectrum in the Improved Opacity Expansion
}

\author{
João Barata ${ }^{a, *}$ \\ ${ }^{a}$ Instituto Galego de Fisica de Altas Enerxias (IGFAE), Universidade de Santiago de Compostela,E-15782 \\ Galicia, Spain
}

E-mail: joaolourenco.henriques@usc.es

Over the last decades, analytical calculations of jet quenching observables have always needed to make a distinction between dense or dilute mediums. Although there are different theoretical formalisms suited for each one of these scenarios, taking into account multiple soft and single hard interactions between the probe and the background under a single approach has proven to be a difficult task. In this talk, we will introduce the Improved Opacity Expansion (IOE), which extends the well established Opacity Expansion framework beyond the hard momentum transfer tail, including the regime captured by the BDMPS-Z/ASW approximation. We will focus on the application of the IOE to the computation of the single gluon medium induced spectrum from a hard parton, which constitutes one of the most important theoretical results in jet quenching theory.

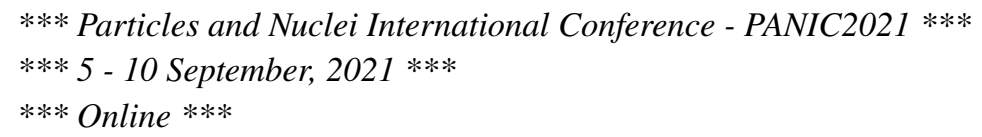

${ }^{*}$ Speaker 


\section{Introduction}

Jet quenching is one of the main signatures for the creation of a quark gluon plasma after the collision of heavy ions at RHIC and the LHCBroadly, it corresponds to the modification of the jet's structure due to the interactions with the underlying hot medium, resulting in the jet's constituents broadening their momentum due to scattering with the medium quasi-particles and the production of induced bremsstrahlung radiation. In the past decades, analytical calculations of these effects have focused on the dilute and dense medium limits, where exact analytic results can be derived.Even though dedicated numerical routines have been designed to study such processes, for many phenomenological applications it would be valuable to have semi-analytical methods to connect these two limits.

In this proceedings, we discuss a novel strategy, dubbed the Improved Opacity Expansion (IOE), which allows to merge the thin and dense medium limits under a single framework. The general strategy of the IOE is first illustrated for the evolution of a single particle in the medium (single particle momentum broadening) and is then used to compute the medium induced gluon spectrum.

\section{A working example: momentum broadening in the IOE approach}

To introduce the IOE formalism [1-5] we consider first the simplest possible observable: single particle momentum broadening. At eikonal accuracy, momentum broadening can be fully characterized by an associated broadening distribution $\mathcal{P}(\boldsymbol{k}, t)$ which gives the probability for a parton to acquire transverse momentum $\boldsymbol{k}$ in the medium as a result of its propagation for a time $t$. In terms of the in-medium elastic collision rate $\gamma_{\mathrm{el}}(\boldsymbol{q}) \sim g^{4} n / \boldsymbol{q}^{4}$, it reads

$$
\frac{\partial \mathcal{P}(\boldsymbol{k}, t)}{\partial t}=C_{R} \int_{\boldsymbol{q}} \gamma_{\mathrm{el}}(\boldsymbol{q})[\mathcal{P}(\boldsymbol{k}-\boldsymbol{q}, t)-\mathcal{P}(\boldsymbol{k}, t)],
$$

where $C_{R}$ is the associated Casimir color factor and $n$ is the density of in-medium scattering centers.

For thin mediums, i.e. when the hard parton interactions a small number of times with the background, $\mathcal{P}$ is dominated by at most a single hard scattering $(\mathrm{SH})$ and it reduces to $\mathcal{P}^{\mathrm{SH}}(\boldsymbol{k}, L)=$ $C_{R} \gamma_{\mathrm{el}}(\boldsymbol{k}) L \propto \frac{\alpha_{s}^{2} C_{R} n L}{\boldsymbol{k}^{4}}$. Conversely, when the medium is dense the probability of scattering becomes of order one and all possible (soft) tree-level gluon exchanges with the medium have to be taken into account. In this regime of multiple soft (MS) scattering with the medium, Eq. (1) can be reduced to a diffusion equation, so that the leading order result reads $\mathcal{P}^{\mathrm{MS}}(\boldsymbol{k}, L)=\frac{4 \pi}{\hat{q} L} \mathrm{e}^{-\frac{\boldsymbol{k}^{2}}{\hat{q} L}}$, where $\hat{q}$ is the jet quenching parameter $\hat{q}=C_{R} \int_{\mu_{*}}^{q_{\max }} \frac{\mathrm{d}^{2} \boldsymbol{q}}{(2 \pi)^{2}} \boldsymbol{q}^{2} \gamma_{\mathrm{el}}(\boldsymbol{q}) \approx 4 \pi \alpha_{s}^{2} C_{R} n \log \frac{q_{\max }^{2}}{\mu_{*}^{2}} \equiv \hat{q}_{0} \log \frac{q_{\max }^{2}}{\mu_{*}^{2}}$. Notice that the in the infrared this integral converges due to the thermal mass $\left(\mu_{*}\right)$ screening and in the ultraviolet the logarithmic divergence must be regulated by a large momentum scale $q_{\text {max }}$. Comparing the above results, it is clear that the SH solution properly describes the expected $1 / \boldsymbol{k}^{4}$ Coulomb form at large momentum, while the MS solution recovers the typical Gaussian solution associated to the resummation of multiple exchanges of soft instantaneous gluons. Although both formulas cover the full range of phenomenological interest for jet quenching when combined, each approximation fails to properly describe the underlying physics in the regime of validity of the other. 


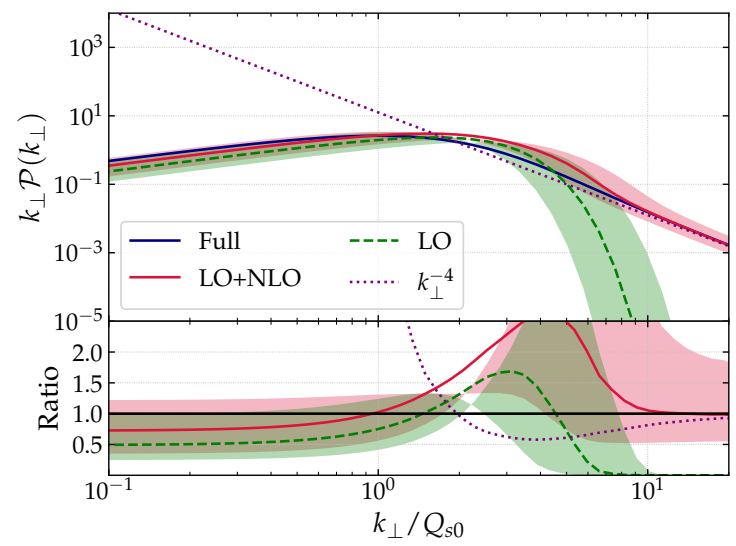

Figure 1: Comparison between the broadening probability distribution in the IOE at LO (dashed, green), at LO+NLO (solid, red) and the exact Gyulassy-Wang modelresult (solid, navy).The medium parameters used in this proceedings are $\hat{q}_{0}=0.16 \mathrm{GeV}^{3}, L=6 \mathrm{fm}$ and $\mu_{*}=0.355 \mathrm{GeV}$. See [5] for more details.

The goal of the IOE expansion is to bridge the gap between the SH and MS regimes. For that we note that

$$
v(\boldsymbol{x})=C_{R} \int_{\boldsymbol{q}} \gamma_{\mathrm{el}}(\boldsymbol{q})\left(1-\mathrm{e}^{i \boldsymbol{q} \cdot \boldsymbol{x}}\right) \propto \boldsymbol{x}^{2} \log \frac{1}{\boldsymbol{x}^{2} \mu_{*}^{2}}+O\left(\boldsymbol{x}^{4} \mu_{*}^{2}\right),
$$

where in the last term we used the fact that at sufficiently small distances the interaction potential is assumed to be of the Coloumb form, and thus any medium model should recover Eq. (2) in that regime. To combine both these regimes, in the IOE, one introduces a matching scale $Q_{b}^{2}$ to rewrite $v(\boldsymbol{x})$, such that

$$
v(\boldsymbol{x}) \propto \boldsymbol{x}^{2} \log \frac{1}{\boldsymbol{x}^{2} \mu_{*}^{2}}=\boldsymbol{x}^{2}\left[\log \frac{Q_{b}^{2}}{\mu_{*}^{2}}+\log \frac{1}{\boldsymbol{x}^{2} Q_{b}^{2}}\right] \propto v_{0}(\boldsymbol{x})+\delta v(\boldsymbol{x}) .
$$

The first term recovers the MS solution (assuming $Q_{b}^{2}=q_{\max }^{2}$ ). Requiring that $Q_{b}^{2} \gg \mu_{*}^{2}$, the second term only becomes dominant at small $\boldsymbol{x}$ (large $\boldsymbol{k}$ ), and thus it can be treated perturbatively. As a result the broadening distribution can be written as [1]

$$
\mathcal{P}^{\mathrm{LO}+\mathrm{NLO}}(\boldsymbol{k}, L)=\frac{4 \pi}{Q_{s}^{2}} \mathrm{e}^{-x}-\frac{4 \pi}{Q_{s}^{2}} \lambda\left\{1-2 \mathrm{e}^{-x}+(1-x)[\operatorname{Ei}(4 x)-\log 4 x]\right\},
$$

where $x=\boldsymbol{k}^{2} / Q_{s}^{2}=\boldsymbol{k}^{2} /\left(\hat{q}_{0} L \log \frac{Q_{b}^{2}}{\mu_{*}^{2}}\right), \lambda=\log ^{-1} \frac{Q_{b}^{2}}{\mu_{*}^{2}} \ll 1$ and $Q_{b}^{2} \equiv Q_{s 0}^{2} \log \frac{Q_{b}^{2}(L)}{\mu_{*}^{2}}$. At large momentum the NLO becomes dominant and we obtain:

$$
\left.\mathcal{P}(\boldsymbol{k}, L)^{\mathrm{NLO}}\right|_{\boldsymbol{k}^{2} \gg Q_{s}^{2}}=4 \pi \frac{Q_{s 0}^{2}}{\boldsymbol{k}^{4}}+O\left(\frac{Q_{s 0}^{4}}{\boldsymbol{k}^{6}}\right) .
$$

In Fig. 1, we compare Eq. (4) to an exact numerical evaluation of $\mathcal{P}$ for a realistic $v(\boldsymbol{x})$ (see $[1,5])$.

\section{Medium induced spectrum in the IOE approach}

In this section we discuss how to apply the IOE approach, illustrated for the broadening case above, to the medium induced (soft) gluon spectrum, differential in the gluon's energy $\omega$ and 
transverse momentum $\boldsymbol{k}$. In general, this can be written as [5]

$$
\begin{gathered}
(2 \pi)^{2} \omega \frac{\mathrm{d} I}{\mathrm{~d} \omega \mathrm{d}^{2} \boldsymbol{k}}=\lim _{\epsilon \rightarrow 0} \frac{2 \bar{\alpha} \pi}{\omega^{2}} \operatorname{Re} \int_{0}^{\infty} \mathrm{d} t_{2} \mathrm{e}^{-\epsilon t_{2}} \int_{0}^{t_{2}} \mathrm{~d} t_{1} \int_{\boldsymbol{x}} \mathrm{e}^{-i \boldsymbol{k} \cdot \boldsymbol{x}} \mathcal{P}\left(\boldsymbol{x}, \infty ; t_{2}\right) \\
\boldsymbol{\partial}_{\boldsymbol{x}} \cdot \boldsymbol{\partial}_{\boldsymbol{y}} \mathcal{K}\left(\boldsymbol{x}, t_{2} ; \boldsymbol{y}, t_{1}\right)_{\boldsymbol{y}=0}-\frac{8 \bar{\alpha} \pi}{k_{\perp}^{2}} .
\end{gathered}
$$

Notice that the previous formula only takes into account purely medium induced radiation off the nascent parton and the adiabatic phase $\mathrm{e}^{-\epsilon t_{2}}$ ensures that interactions are properly turned-off at asymptotically large times. In the present form, one observes that the full spectrum can be written as the broadening of a gluon final state in the time interval $\left(t_{2}, \infty\right)$, being preceded by its production via an effective kernel $\mathcal{K}$ during $\left(t_{1}, t_{2}\right)$. The treatment of $\mathcal{P}$ follows the discussion in the previous section.Truncating at first order in $\delta v$, we can write Eq. (6) to first order in the IOE as

$$
\frac{\mathrm{d} I}{\mathrm{~d} \omega \mathrm{d}^{2} \boldsymbol{k}}=\frac{\mathrm{d} I^{\mathrm{LO}}}{\mathrm{d} \omega \mathrm{d}^{2} \boldsymbol{k}}+\frac{\mathrm{d} I^{\mathrm{NLO}}}{\mathrm{d} \omega \mathrm{d}^{2} \boldsymbol{k}}+O\left(\delta v^{2}\right)
$$

The first term corresponds to the MS result with a matching scale $Q_{r}$ given by $Q_{r}^{2}=\sqrt{\hat{q}_{0} \omega \log \frac{Q_{r}^{2}}{\mu_{*}^{2}}}$ where $\sqrt{\hat{q}_{0} \omega}$ is the typical transverse momentum accumulated by the gluon during the emission process. The NLO term takes into account the leading order corrections in $\delta v$. Explicitly these it can be written as

$$
\begin{aligned}
(2 \pi)^{2} \omega \frac{\mathrm{d} I^{\mathrm{NLO}}}{\mathrm{d} \omega \mathrm{d}^{2} \boldsymbol{k}} & =\frac{2 \bar{\alpha} \pi}{\omega^{2}} \operatorname{Re} \int_{0}^{\infty} \mathrm{d} t_{2} \mathrm{e}^{-\epsilon t_{2}} \int_{0}^{t_{2}} \mathrm{~d} t_{1} \int_{\boldsymbol{x}} \mathrm{e}^{-i \boldsymbol{k} \cdot \boldsymbol{x}}\left[\mathcal{P}^{\mathrm{LO}}\left(\boldsymbol{x}, \infty ; t_{2}\right) \boldsymbol{\partial}_{\boldsymbol{x}} \cdot \boldsymbol{\partial}_{\boldsymbol{y}} \mathcal{K}^{\mathrm{NLO}}\left(\boldsymbol{x}, t_{2} ; \boldsymbol{y}, t_{1}\right)_{\boldsymbol{y}=0}\right. \\
& \left.+\mathcal{P}^{\mathrm{NLO}}\left(\boldsymbol{x}, \infty ; t_{2}\right) \boldsymbol{\partial}_{\boldsymbol{x}} \cdot \boldsymbol{\partial}_{\boldsymbol{y}} \mathcal{K}^{\mathrm{LO}}\left(\boldsymbol{x}, t_{2} ; \boldsymbol{y}, t_{1}\right)_{\boldsymbol{y}=0}\right]
\end{aligned}
$$

where we used $\mathcal{P}^{\mathrm{NLO}}(\boldsymbol{x}, \infty ; t)=-\mathcal{P}^{\mathrm{LO}}(\boldsymbol{x}, \infty ; t) \int_{t}^{\infty} \mathrm{d} s \delta v(\boldsymbol{x}, s)$. Notice that the first order correction to the MS result given in Eq. (8) has a term related to modification of the emission kernel (followed by the usual Gaussian broadening), and a term describing the production of a gluon in the MS approximation followed by broadening dominated by single hard scattering contribution. Performing a slightly evolved calculation, it results that (see details in [5]) $(2 \pi)^{2} \omega \frac{\mathrm{d} I^{\mathrm{NLO}}}{\mathrm{d} \omega \mathrm{d}^{2} \boldsymbol{k}} \approx \frac{8 \pi \bar{\alpha} \hat{q}_{0} L}{\boldsymbol{k}^{4}}\left[3 \gamma_{E}-4+\log \left(\frac{\boldsymbol{k}^{2}}{4 Q_{b}^{2}}\right)+\log \left(\frac{\boldsymbol{k}^{2} L}{2 \omega}\right)\right]$. Unlike in Eq. (5), it seems that there is still a dependence on the matching scale $Q_{b}$. However, this dependence vanishes once LO subleading corrections are taken into account. The full spectrum at this accuracy exactly gives $(2 \pi)^{2} \omega \frac{\mathrm{d} I^{\mathrm{LO}+\mathrm{NLO}}}{\mathrm{d} \omega \mathrm{d}^{2} \boldsymbol{k}}=\frac{8 \pi \bar{\alpha} \hat{q}_{0} L}{\boldsymbol{k}^{4}}\left[3 \gamma_{E}-4+\log \left(\frac{\boldsymbol{k}^{2}}{4 \mu_{*}^{2}}\right)+\log \left(\frac{\boldsymbol{k}^{2} L}{2 \omega}\right)\right]=(2 \pi)^{2} \omega \frac{\mathrm{d} I^{\mathrm{SH}}}{\mathrm{d} \omega \mathrm{d}^{2} \boldsymbol{k}}$, as expected.

The expressions given above can be further simplified; a numerical code to compute them can be found in [6] with comparable computational cost with respect to previous implementations of the MS result. To illustrate the accuracy of the IOE approach, in Fig. 2 we show the differential spectrum for two gluon frequencies $\left(\omega_{c 0} \equiv \hat{q}_{0} L^{2}\right)$.

\section{Conclusion and Outlook}

We have illustrated how to extend the Improved Opacity Expansion to the case of the double differential medium induced gluon spectrum. Thus, the IOE formalism allows for the first time to include the effects of both multiple soft and single hard scattering in the medium under a single framework. 

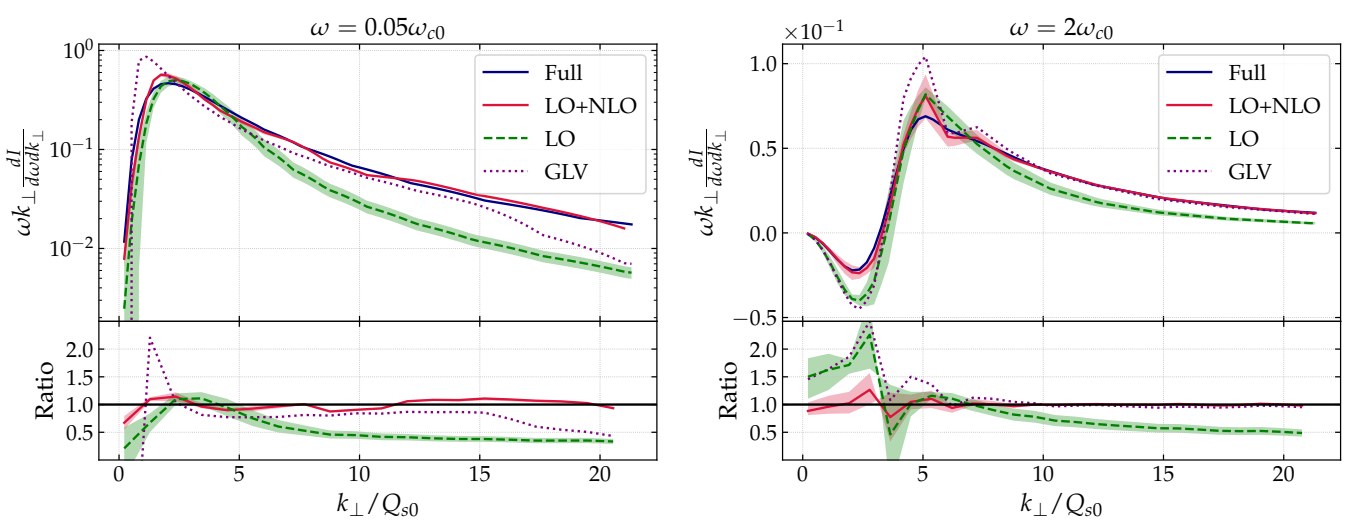

Figure 2: Comparison between the SH (GLV) spectrum (dotted, purple), the LO result (dashed, green), the IOE at LO+NLO (solid, red) and the all-order spectrum (solid, navy) as computed in [7] for two gluon frequencies: $\omega=0.05 \omega_{c 0}$ (left) and $\omega=2 \omega_{c 0}$ (right). The ratio to the full solution is presented in the bottom panels. The uncertainty band arises from variations in the matching scales $Q_{b}$ and $Q_{r}$.

\section{Acknowledgements}

J.B. is supported by Ministerio de Ciencia e Innovacion of Spain under project FPA201783814-P; Unidad de Excelencia Maria de Maetzu under project MDM-2016-0692; European research Council project ERC-2018-ADG-835105 YoctoLHC; and Xunta de Galicia (Conselleria de Educacion) and FEDER.

\section{References}

[1] J. Barata, Y. Mehtar-Tani, A. Soto-Ontoso, K. Tywoniuk, Revisiting transverse momentum broadening in dense QCD media, Phys. Rev. D 104 (5) (2021) 054047. arXiv:2009.13667, doi: 10.1103/PhysRevD. 104.054047.

[2] Y. Mehtar-Tani, Gluon bremsstrahlung in finite media beyond multiple soft scattering approximation, JHEP 07 (2019) 057. arXiv: 1903.00506, doi : 10 . 1007/JHEP07 (2019) 057.

[3] Y. Mehtar-Tani, K. Tywoniuk, Improved opacity expansion for medium-induced parton splitting, JHEP 06 (2020) 187. arXiv: 1910.02032, doi : 10 . 1007/JHEP06(2020) 187.

[4] J. Barata, Y. Mehtar-Tani, Improved opacity expansion at NNLO for medium induced gluon radiation, JHEP 10 (2020) 176. arXiv:2004.02323, doi : 10.1007/JHEP10(2020) 176.

[5] J. Barata, Y. Mehtar-Tani, A. Soto-Ontoso, K. Tywoniuk, Medium-induced radiative kernel with the Improved Opacity Expansion (6 2021). arXiv:2106.07402, doi:10.1007/ JHEP09 (2021) 153.

[6] https://github.com/albaontoso/IOE-ktspectrum.

[7] C. Andres, L. Apolinário, F. Dominguez, Medium-induced gluon radiation with full resummation of multiple scatterings for realistic parton-medium interactions, JHEP 07 (2020) 114. arXiv:2002 .01517, doi:10.1007/JHEP07 (2020) 114 . 